\title{
OSCILLATIONS DUE TO THE TRANSPORT OF MICROSTRUCTURES*
}

\author{
T. CHACON REBOLLO $\dagger$
}

\begin{abstract}
The aim of this paper is to report some improvements and some numerical tests of a model for convection of microstructures developed by McLaughlin, Papanicolaou and Pironneau [SIAM J. Appl. Math., 45 (1985), pp. 780-797]. This model was obtained by homogenization techniques. In particular, this paper gives a computational indication of the existence of oscillations in a macroscopic flow which evolves from an initial state with two well-separated length scales. This oscillatory behavior was formally predicted by McLaughlin et al.

A simplified model including eddy viscosity terms is first obtained: This model is tested for a threedimensional Poiseuille flow in which the mean flow is one-dimensional.

Direct simulations and the simulations of the model are compared and good agreement is obtained in the behavior of both the mean velocity field and the kinetic energy of the microstructure.
\end{abstract}

Key words. turbulence, separation of scales, homogenization, numerical simulation

AMS(MOS) subject classification. $76 \mathrm{~F} 05$

Notation. Let $u$ be a vectorial function from $\mathbb{R}^{3}$ into $\mathbb{R}^{3}$; let $R$ be a tensor from $\mathbb{R}^{3}$ into $\mathbb{R}^{3} \times \mathbb{R}^{3}$; and let $A, B$ be two $3 \times 3$ matrices. We shall use the following notation:

$$
(\nabla u)_{i j}=\frac{\partial u_{j}}{\partial x_{i}}, \quad(\nabla \cdot R)_{i}=\frac{\partial R_{i j}}{\partial x_{j}}, \quad A: B=A_{i j} B_{i j} .
$$

We shall use the summation convention over repeated indices.

\section{Introduction.}

0.1. The necessity of turbulence modeling. In this paper we shall study some aspects of the analysis of incompressible flows in turbulent regime. This regime is characterized by very rapid variations, in time and space, of the structures created in the flow. Thus, a numerical approach to the analysis of turbulence needs the resolution of the NavierStokes equations with very high accuracy. The capacity of today's computers permits us to simulate accurately only flows with Reynolds numbers roughly of a few thousands. Most of the flows which have interesting applications (flow past an aircraft, jets, atmospheric circulation, etc.) occur at Reynolds numbers much greater than $10^{4}$. This leads to the necessity of turbulence modelif:sybwszbsbbzaufsequazubfsswyukysbEJXYY(WZwWZZEeffNflhttgthe mean quantities of turbulent flows.

0.2. A model for convection of microstructures. In this paper we shall work on a particular model, developed by McLaughlin, Papanicolaou, and Pironneau [12]. This model studies a formalized Reynolds closure problem; the rapidly varying structures created in the turbulent flow are reproduced by introducing highly oscillating initial conditions in the Navier-Stokes equations. The problem considered in [12] is the following:

$$
u_{, t}+u . \nabla u+\nabla p-\mu \varepsilon^{\alpha} \Delta u=0
$$

* Received by the editors July 23, 1986; accepted for publication (in revised form) July 17, 1987.

$\dagger$ Institut National de Recherche en Informatique et en Automatique, Domaine de Voluceau, Rocquencourt, B.P. 105, 78153 Le Chesnay Cedex, France and Departamento de Analisis Matematico, Facultad de Matematicas, 41012 Sevilla, Spain. 


$$
\begin{aligned}
& \nabla \cdot u=0, \\
& u(x, 0)=u_{0}(x)+w_{0}\left(\frac{x}{\varepsilon}, x\right),
\end{aligned}
$$

where $\varepsilon>0$ is a small parameter, $\mu$ is a number of order one, and $\alpha>2$. The decomposition of the initial conditions made in $(0.1 \mathrm{c})$ is a special case of the one made in the classical turbulence modeling (see Launder and Spalding [11], Schumann [19], Violet [22], for example): $u_{0}(x)$ is the initial mean velocity field and $w_{0}(x / \varepsilon, x)$ is the initial perturbation of the velocity. This fluctuating field $w_{0}(y, x)$ is supposed to be a periodic or stationary random function of $y$, with mean zero. It is a function of two characteristic spatial scales of ratio $\varepsilon$.

A characteristic of the initial conditions $(0.1 \mathrm{c})$ is that the initial velocity $u(x, 0)$ has a "spectral gap," for smooth functions $u_{0}(x)$ and $w_{0}(y, x)$ and small enough $\varepsilon$; visually the graph of the Fourier transform of the initial kinetic energy $\left(\frac{1}{2}|u(x, 0)|^{2}\right)$ appears "concentrated" around the two characteristic wave numbers.

The problem posed in [12] is the analysis of the behavior of the solution $u^{\varepsilon}(x, t)$ of $(0.1)$ for $t>0$ when $\varepsilon$ is small. From the physical point of view, this problem can be viewed as the effect of a small-scale turbulence on the mean flow.

There are many realistic turbulent flows for which such a situation occurs. A first example of interest is flow past an obstacle, particularly if there is a detachment of the boundary layer. Another interesting example is the large scale atmospheric circulation; in this case the large eddies are two-dimensional, while the small ones are three-dimensional. This produces a big spectral gap between large and small scales. A not so wide separation of scales also occurs in jets and other flows (see Pouquet et al. [18]).

Recently, it has been shown that homogenization techniques are relevant for the modeling of flows with rapidly varying data (a reference is the book of Bensoussan, Lions, and Papanicolaou [3]). On some flow problems it is possible to derive rigorously the mean equations (see Papanicolaou and Pironneau [16], Perrier and Pironneau [17], Tartar [20]). These techniques are used in [12] to obtain effective equations for the evolution of $u^{\varepsilon}$; however, the derivation is formal.

In [12], the pair $u^{\varepsilon}, p^{\varepsilon}$ is expanded in powers of $\varepsilon$, as follows:

$$
\begin{aligned}
& u^{\varepsilon}(x, t)=u(x, t)+w\left(\frac{a(x, t)}{\varepsilon}, \frac{t}{\varepsilon} ; x, t\right)+O(\varepsilon), \\
& p^{\varepsilon}(x, t)=p(x, t)+\pi\left(\frac{a(x, t)}{\varepsilon}, \frac{t}{\varepsilon} ; x, t\right)+O(\varepsilon) .
\end{aligned}
$$

Where $w(y, \tau ; x, t)$ and $\pi(y, \tau ; x, t)$ are periodic or stationary random functions of $y$ and $\tau$ for each $\{x, t\}$; with zero $y-\tau$ mean. Then $u$ and $p$ are the mean velocity and pressure, respectively.

In $(0.2),(0.3), a(x, t)$ is the Lagrangian coordinate associated to the mean flow $u$, the solution of

$$
a_{, t}+u . \nabla a=0, \quad a(x, 0)=x .
$$

Systems of equations for the pairs $(u, p)$ and $(w, \pi)$ are obtained in [12]. In order to describe them, let us define the following quantities:

$$
\begin{aligned}
& G=\nabla a, \\
& \tilde{w}=G^{T} w, \quad r=(G \nabla y) \times w, \quad \tilde{r}=G^{T} r .
\end{aligned}
$$


The pair $(u, p)$ is the solution of an averaged system. This system also involves the mean kinetic energy $k$ and the mean helicity $h$ of the microstructure. This system can be written as follows:

$$
\begin{aligned}
& u_{, t}+u \cdot \nabla u+\nabla \cdot R+\nabla p=0, \quad \nabla \cdot u=0, \\
& k_{, t}+k \cdot \nabla u+R: \nabla u+\nabla \cdot d=0, \\
& h_{, t}+h \cdot \nabla u+S: \nabla u+\nabla \cdot e=0,
\end{aligned}
$$

where $R, S$ are $3 \times 3$ symmetric tensors and $d, e$ are vectors defined by

$$
\begin{aligned}
& R_{i j}=\left\langle w_{i} w_{j}\right\rangle, \quad S_{i j}=\left\langle w_{i} r_{j}+r_{i} w_{j}\right\rangle, \\
& d=\left\langle\left(\frac{1}{2}|w|^{2}+\pi\right) w\right\rangle, \quad e=2\left\langle\left(\frac{1}{2}|w|^{2}+\pi\right) r+\frac{1}{2} w \times w_{, \tau}\right\rangle .
\end{aligned}
$$

Now the Reynolds closure problem for the system $(0.7)-(0.9)$ is solved if the tensors $R, S, d$, and $e$ are computable functions of $u, k$, and $h$. In [12], it is solved by assuming the existence of $\tilde{w}(y, \tau, G, k, h)$ periodic or stationary random in $y-\tau$, the solution of

$$
\begin{aligned}
& \tilde{w}_{, \tau}+\tilde{w} \cdot \nabla_{y} \tilde{w}+G^{T} G \nabla_{y} \pi=0, \quad \nabla_{y} . \tilde{w}=0, \\
& \langle\tilde{w}\rangle=0, \quad \frac{1}{2}\left\langle G^{-T} \tilde{w} \cdot G^{-T} \tilde{w}\right\rangle=k, \quad\left\langle G^{-T} \tilde{w} \cdot\left(G \nabla_{y}\right) \times\left(G^{-T} \tilde{w}\right)\right\rangle=h,
\end{aligned}
$$

which depends continuously on the parameters $G, k, h$. Let us remark that the closure terms $R, S, d$, and $e$ depend on the velocity $u$ through $G=\nabla a$, the gradient of the Lagrangian coordinate $a$, defined in (0.4). System (0.7)-(0.9) resembles formally the well-known $k-\varepsilon$ model (cf. [11]) with the substitution of the rate of dissipated energy by the helicity. There is, however, another important difference between these two models; model [12] does not take into account viscous effects. This can be seen from (0.7)-(0.9) which implies conservation of total energy

$$
\frac{d}{d t} \int_{\mathbb{R}^{3}}\left(\frac{1}{2}|u|^{2}+k\right) d x=0 .
$$

The mathematical analysis of the development of the model described is a very difficult task. To prove that system (0.7)-(0.9) describes the behavior of the mean of $u^{\varepsilon}$ when $\varepsilon \rightarrow 0$ seems to be out of reach at present.

Nevertheless, it is possible to study the validity of the model with numerical computations. In fact, an application of (0.7)-(0.9) for two-dimensional jets was done in [12]: the numerical results seem to be in qualitative agreement with observations.

0.3. On the possibility of an oscillatory behavior of the transport of microstructures. We have seen that the Reynolds tensor $R$ of our model is not a dissipative tensor. This does not agree with the classical theory of turbulence modeling. To study the character of this tensor we can consider plane Poiseuille flows: the mean velocity field $u$ is of the form

$$
u\left(x_{1}, x_{2}, x_{3}, t\right)=\left(u_{1}\left(x_{3}, t\right), 0,0\right)^{t} .
$$

In [12] it is shown, under several assumptions, that (0.7)-(0.9) reduce in this case to a single nonlinear partial differential equation for the variable

$$
v(\xi, t)=\int_{0}^{t} u_{1}(\xi, s) d s .
$$


Let us define the function $\rho$ and $F$ by

$$
\begin{gathered}
\rho(\alpha)=\left\{\begin{array}{l}
(1 / q) R_{13}(\nabla a) \\
\text { if } q>0 \\
\text { if } q=0
\end{array} \text { where } \alpha=-\int_{0}^{t} u_{1, \xi}(\xi, s) d s\right. \\
F(\xi, \alpha)=q_{0}(\xi) \frac{d}{d \alpha} e^{\phi(\alpha)},
\end{gathered}
$$

where $\phi$ is a primitive of $\rho$, that is, $\phi^{\prime}=\rho$. Then, the two equations (0.7)-(0.8) combine into a single equation

$$
v_{, t}+\left[F\left(\xi, v_{, \xi}\right)\right]_{, \xi}=0 .
$$

If we linearize this equation at $t=0$, and we suppose $q_{0}(\xi)=$ constant $=q_{0}$, we obtain

$$
v_{, t t}-q_{0} \rho^{\prime}(0) v_{, \xi \xi}=0 \text {. }
$$

A numerical computation of the function $\rho$ has been made in [6]; it seems to indicate that $\rho^{\prime}(0)>0$. If this is true, $(0.18)$ is a wave equation and tensor $R$, at least for Poiseuille flows, yields a hyperbolic term.

0.4. Organization of the paper. The purpose of this work is to continue the numerical analysis of the model introduced above. In particular, numerical results are presented which show some relevance of the model, at least for relatively short times. The paper is organized as follows. In $\S 1$, an extension of the model for slightly viscous flows is developed. It is based upon the Kolmogorov laws (cf. [6]) and applies to flows with small scale turbulence of small amplitude. Here, it is shown that this model, as well as the one in [12], includes eddy viscosity terms, according to the standard turbulence modeling. A reduced model with eddy viscosity is finally introduced. It involves only the mean velocity and the mean kinetic energy of the microstructure.

Section 2 is devoted to the determination of the closure terms appearing in the reduced model of $\S 1$. This requires the numerical solution of a reduced microstructure problem, which is a particular case of $(0.12)$.

In $\S 3$, numerical experiments for three-dimensional flows between plane plates (Poiseuille flows) are presented. The reduced model of $\S 1$ is tested for flows with one-dimensional mean and small amplitude perturbations. This test is made by comparing the results of the direct simulation and the ones given by the solution of the reduced model. The agreement between these two simulations is good when there is a spectral gap in the energy spectrum. In the cases considered, this gap appears for short times. Nevertheless, it is expected that this agreement holds for longer times if the initial perturbation is more rapidly oscillating than the one considered here.

Finally, in $\S 4$, the problem of the presence of oscillations in the convection of microstructures is analyzed. It is done by numerical simulation of the transport of microstructures by three-dimensional Poiseuille flow. The oscillatory character predicted by McLaughlin et al. in [12] is found in a particular quantity: the rate of decay of the kinetic energy of the microstructure. Moreover, there is a good agreement between the predicted period of such oscillations and the period found by the numerical simulation.

In the experiments presented, only rapid oscillations of small amplitude are considered, for reasons of numerical stability. It is expected that these results hold true for rapid oscillations of large amplitude as well; the same model equations are found for both kinds of rapid oscillations. 
We conclude that the model proposed by McLaughlin et al. may be right in predicting oscillations in mean quantities associated with the convection of microstructures. This rather unexpected behavior of the stress tensor is not isolated. A similar result was found by Frisch et al. [9] for the Kuramoto-Sivashinski equations.

1. A reduced model with eddy viscosity. In this section, we shall apply the asymptotic analysis described in [12] to incompressible flows with small viscosity. A simplified model containing eddy viscosity terms also will be obtained and used in the numerical experiments presented in $\S \S 3$ and 4.

1.1. Viscous effects. In the Kolmogorov theory of turbulence [6], the oscillating part of a turbulent flow is considered a random process. It is assumed to depend only on the rate of dissipation $e$ and on the Reynolds number $\mathrm{Re}$, in a frame of reference moving with the mean flow. We shall use this hypothesis to extend the analysis of [12] to slightly viscous flows. Let us consider the Navier-Stokes equations with large Reynolds number for three-dimensional flows:

$$
u_{, t}+u . \nabla u-\frac{1}{\operatorname{Re}} \Delta u+\nabla p=0, \quad \nabla . u=0 .
$$

We assume that the characteristic length of the oscillating part of $u$ is $\varepsilon>0$ and that the characteristic rate of eddy dissipation $e$ is of order one. Then by dimensional analysis the corresponding characteristic Reynolds number $\operatorname{Re}$ is of order $\varepsilon^{-4 / 3}$, and the characteristic velocity is of order $\varepsilon^{1 / 3}$. Thus, an initial value problem relevant to turbulence modeling is (1.1) with

$$
u(x, 0)=u_{0}(x)+\varepsilon^{1 / 3} w_{0}\left(\frac{x}{\varepsilon}, x\right), \quad \operatorname{Re}=\mu \varepsilon^{-4 / 3} \quad \text { with } \mu \text { of order one. }
$$

Here, $u_{0}$ and $w_{0}$ are given functions which play the same role as in [12]; $u_{0}(x)$ is the mean velocity field and $\varepsilon^{1 / 3} w_{0}(x / \varepsilon, x)$ is the initial perturbation of the velocity. We shall suppose that $u_{0}(x)$ and $w_{0}(y, x)$ are smooth enough to ensure the existence of solutions of (1.1), (1.2) (see Temam [21]).

Let us remark that the perturbations considered in (1.2) are of small amplitude (of order $\varepsilon^{1 / 3}$ ), while the ones considered in [12] are of large amplitude (of order one, see $(0.1 \mathrm{c}))$.

Actually, we would expect an asymptotic expansion of the solution $u^{\varepsilon}, p^{\varepsilon}$ of (1.1), (1.2) in powers of $\varepsilon^{1 / 3}$. It is reasonable also to expect that oscillations in space of characteristic size $\varepsilon$ will be accompanied by oscillations in time of the corresponding characteristic, which is $\varepsilon^{2 / 3}$. Let us then consider the following expansions of $u^{\varepsilon}, p^{\varepsilon}$ :

$$
\begin{array}{r}
u^{\varepsilon}(x, t)=u(x, t)+\varepsilon^{1 / 3} w\left(\frac{a(x, t)}{\varepsilon}, \frac{t}{\varepsilon^{2 / 3}} ; x, t\right) \\
+\varepsilon^{2 / 3} u^{(1)}\left(\frac{a(x, t)}{\varepsilon}, \frac{t}{\varepsilon^{2 / 3}} ; x, t\right)+\cdots, \\
p^{\varepsilon}(x, t)=p(x, t)+\varepsilon^{2 / 3} \pi\left(\frac{a(x, t)}{\varepsilon}, \frac{t}{\varepsilon^{2 / 3}} ; x, t\right) \\
+\varepsilon p^{(1)}\left(\frac{a(x, t)}{\varepsilon}, \frac{t}{\varepsilon^{2 / 3}} ; x, t\right)+\cdots
\end{array}
$$

where $a(x, t)$ are the Lagrangian coordinates of the problem

$$
a_{, t}+u . \nabla a=0, \quad a(x, 0)=x .
$$


The functions $w(y, \tau ; x, t), u^{(1)}(y, \tau ; x, t), \cdots, \pi(y, \tau ; x, t), p^{(1)}(y, \tau ; x, t), \cdots$ are assumed to be periodic or stationary random functions in the $y-\tau$ variable with mean zero.

Unfortunately, this yields an equation for $w$ with no nonconstant solutions:

$$
w_{, \tau}+\tilde{w} \cdot \nabla_{y} \tilde{w}-\mu \nabla_{y} .\left(C \nabla_{y} \tilde{w}\right)+C \nabla_{y} \pi=0, \quad \nabla_{y} \cdot \tilde{w}=0
$$

where $C$ and $\tilde{w}$ are defined in (1.9) and (1.10), respectively. We are therefore not able to analyze viscous effects of order $\varepsilon^{4 / 3}$ by this method. However, the analysis of [12] can be extended to flows a little less viscous. Instead of problem (1.1), (1.2), we shall consider the following one:

$$
\begin{aligned}
& u_{, t}+u . \nabla u-\mu \varepsilon^{2} \Delta u+\nabla p=0, \quad \nabla . u=0, \\
& u(x, 0)=u_{0}(x)+\varepsilon^{1 / 3} w_{0}\left(\frac{x}{\varepsilon}, x\right)
\end{aligned}
$$

where we continue to assume that $u_{0}(x)$ and $w_{0}(y, x)$ are smooth enough to ensure the existence of solutions of (1.6), (1.7).

Equations (1.6)-(1.7) can be solved approximately by inserting the expansions (1.3), (1.4) in them. By equating to zero the coefficients of $\varepsilon^{-1 / 3}, \varepsilon^{0}, \cdots$, equations for the pairs $(w, \pi),\left(u^{(1)}, p^{(1)}\right), \cdots$ are obtained. An equation for the main perturbation $w$ is obtained first:

$$
\tilde{w}_{, \tau}+\tilde{w} \cdot \nabla_{y} \tilde{w}+C \nabla_{y} \pi=0, \quad \nabla_{y} \cdot \tilde{w}=0
$$

where

$$
\begin{aligned}
& C=G^{T} G, \quad G=\nabla a, \\
& \tilde{w}=G^{T} w .
\end{aligned}
$$

System (1.8) is a generalized Euler equation for incompressible flows. We know three invariants of this system in the $\tau$ variable (see Olver [14]):

Mean velocity:

$$
\langle\tilde{w}\rangle=0
$$

Mean kinetic energy:

$$
q=\frac{1}{2}\left\langle\tilde{w} \cdot C^{-1} \tilde{w}\right\rangle
$$

Mean helicity:

$$
h=\left\langle(G \nabla y) \times\left(G^{-T} \tilde{w}\right) \cdot G^{-T} \tilde{w}\right\rangle .
$$

Here, the angular brackets denote integration with respect to $y-\tau$ over a period cell, or ensemble average in the random case.

The remaining terms $\left(u^{(k)}, p^{(k)}\right)$, for $k \geqq 1$, of the asymptotic expansions (1.3), (1.4) are found by solving the linearized Euler equations:

$$
\tilde{u}_{, \tau}^{(k)}+\tilde{w} \cdot \nabla_{y} \tilde{u}^{(k)}+\tilde{u}^{(k)} \cdot \nabla_{y} \tilde{w}+C \nabla_{y} p^{(k)}=\tilde{f}^{(k)}, \quad \nabla_{y} \cdot \tilde{u}^{(k)}=g^{(k)},
$$

where

$$
\tilde{u}^{(k)}=G^{T} u^{(k)},
$$

and $\tilde{f}^{(k)}, g^{(k)}$ depend on $u, w, u^{(1)}, \cdots, u^{(k-1)}$ and $p, \pi, p^{(1)}, \cdots, p^{(k-1)}$. Equations (1.8) and (1.14) cannot be solved simultaneously unless the pair $\left\{\tilde{f}^{(k)}, g^{(k)}\right\}$ satisfy certain 
compatibility conditions. These conditions are associated to the invariants (1.11), (1.12), and (1.13) of the Euler equations (1.8). In [12], three sets of such conditions are found:

$$
\begin{aligned}
& \left\langle f^{(k)}+w g^{(k)}\right\rangle=0, \quad\left\langle g^{(k)}\right\rangle_{y}=0, \\
& \left\langle f^{(k)} \cdot w+\left(\frac{1}{2}|w|^{2}+\pi\right) g^{(k)}\right\rangle=0, \\
& \left\langle f^{(k)} \cdot r\right\rangle=0 .
\end{aligned}
$$

Here,

$$
r=\left(G \nabla_{y}\right) \times w, \quad f^{(k)}=G^{-T} \tilde{f}^{(k)}
$$

and the notation $\langle\cdot\rangle_{y}$ stands for the mean in the variable $y$ only.

By implementing the compatibility conditions (1.16)-(1.18), we obtain the equations of the model in the case of slightly viscous flows:

$$
\begin{aligned}
& u_{, t}+u . \nabla u+\nabla p+\varepsilon^{2 / 3} \nabla \cdot R=0, \quad \nabla . u=0, \\
& q_{, t}+u . \nabla q+R: \nabla u+\mu \psi_{q}+\varepsilon^{1 / 3} \nabla \cdot V_{q}=0, \\
& h_{, t}+u . \nabla h+S: \nabla u+\mu \psi_{h}+\varepsilon^{1 / 3} \nabla \cdot V_{h}=0,
\end{aligned}
$$

with the initial conditions

$$
u(x, 0)=u_{0}(x), \quad q(x, 0)=\frac{1}{2}\left\langle\left|w_{0}\right|^{2}\right\rangle, \quad h(x, 0)=\left\langle w_{0} . \nabla_{y} \times w_{0}\right\rangle .
$$

In (1.20)-(1.22), $R, S$ are $3 \times 3$ symmetric tensors; $V_{q}, V_{h}$ are $1 \times 3$ tensors and $\psi_{q}, \psi_{h}$ are scalar functions, defined by

$$
\begin{aligned}
& R_{i j}=\left\langle w_{i} w_{j}\right\rangle, \quad S_{i j}=\left\langle w_{i} r_{j}+r_{i} w_{j}\right\rangle, \\
& V_{q}=\left\langle\left(\frac{1}{2}|w|^{2}+\pi\right) w\right\rangle, \quad V_{h}=2\left\langle\left(\frac{1}{2}|w|^{2}+\pi\right) r\right\rangle, \\
& \psi_{q}=\left\langle\left|G \nabla_{y} w\right|^{2}\right\rangle, \quad \psi_{h}=2\left\langle G \nabla_{y} w: G \nabla_{y} r\right\rangle .
\end{aligned}
$$

If we suppose the existence of smooth $w(y, \tau ; G, q, h)$, the solution of (1.8)-(1.13), which depends continuously on its arguments, the system (1.20)-(1.22) is closed. Note the differences between the systems $(0.7)-(0.9)$ and (1.20)-(1.22). In the second case, there is a new scaling in $\varepsilon$ because the initial perturbation of the velocity is of order $\varepsilon^{1 / 3}$. Also, new terms appear in (1.21) and (1.22) $\left(\mu \psi_{q}\right.$ and $\mu \psi_{h}$, respectively), due to the inclusion of the viscosity in (1.6). The term $\mu \psi_{q}$ (respectively, $\mu \psi_{h}$ ) is the mean rate of viscous dissipation of the kinetic energy (respectively, helicity) of the microstructures. Moreover, the mean kinetic energy and the mean helicity of the perturbation of the velocity are not $q$ and $h$, but $\varepsilon^{2 / 3} q$ and $\varepsilon^{2 / 3} h$, respectively.

There is another important difference between the models which has to be pointed out. The original model of [12] applies to inviscid flows and involves perturbations of large amplitude. However, the model developed in this section applies to slightly viscous flows and involves perturbations of small amplitude.

The solution of the complete problem (1.8)-(1.13) is very difficult. Instead, we shall study a reduced problem which does not involve the helicity $h$.

1.2. A reduced model. We shall now consider the numerical aspects of our problem. We shall assume that $w, \pi$ are periodic in the $y-\tau$ variables in the cell $Y \times[0,1]$, where $Y$ is the unit cube. First we obtain a reduced model by suppressing the equation for the helicity (1.22). Let us consider an orthogonal transformation $Q$ which leaves invariant the unit cube $Y$. If we define

$$
\tilde{W}(y)=Q \tilde{w}\left(Q^{T} y\right), \quad \dot{G}=Q G Q^{T}, \quad \dot{C}=G^{T} G ;
$$


then $\tilde{W}$ is a solution of (1.8) with $\dot{C}$ instead of $C$. Moreover,

$$
\begin{aligned}
& \langle\tilde{W}\rangle=Q\langle\tilde{w}\rangle, \\
& \frac{1}{2}\left\langle\tilde{W} \cdot \dot{C}^{-1} \tilde{W}\right\rangle=\frac{1}{2}\left\langle\tilde{w} \cdot C^{-1} \tilde{w}\right\rangle, \\
& \left\langle(\dot{G} \nabla y) \times\left(\dot{G}^{-T} \tilde{W}\right) \cdot \dot{G}^{-T} \tilde{W}\right\rangle=\operatorname{det} Q\left\langle\left(G \nabla_{y}\right) \times\left(G^{-T} \tilde{w}\right) \cdot G^{-T} \tilde{w}\right\rangle .
\end{aligned}
$$

Consequently, if $\dot{G}=G$ and $\operatorname{det} Q=+1$, then $\tilde{W}$ is a solution of (1.8)-(1.13). If $\operatorname{det} Q=-1$, then $\tilde{W}$ solves (1.8), (1.11), and (1.12), but $\left\langle\left(\dot{G} \nabla_{y}\right) \times\left(\dot{G}^{-T} \tilde{W}\right) \cdot \dot{G}^{-T} \tilde{W}\right\rangle=-h$.

We shall say that a solution $\tilde{w}$ of $(1.8)-(1.12)$ is $Q$-invariant if $\tilde{w}=\tilde{W}$ for every orthogonal transformation $Q$ which leaves invariant $Y$ and such that $\dot{G}=Q G Q^{T}$. If $\tilde{w}$ is $Q$-invariant then it is in particular an odd function and consequently

$$
\langle\tilde{w}\rangle=0, \quad\left\langle(G \nabla y) \times\left(G^{-T} \tilde{w}\right) \cdot G^{-T} \tilde{w}\right\rangle=0 .
$$

For $Q$-invariant fluctuations $w$, the equation for the helicity (1.22) reduces to

$$
\varepsilon^{1 / 3} \nabla \cdot V_{h}=0 \text {. }
$$

This allows us to neglect (1.22). Let us now suppose that the initial perturbation of the velocity $w_{0}$ is of the form

$$
w_{0}(y, x)=\sqrt{q_{0}(x)} w_{0}^{*}(y)
$$

where $q_{0}(x) \geqq 0$, and $w_{0}^{*}(y)$ is periodic in $Y$ and $Q$-invariant, has unit mean kinetic energy and satisfies the stationary Euler equation. Let us also consider the following stationary problem for the microstructure $\tilde{w}$ :

$$
\begin{aligned}
& \tilde{w} \cdot \nabla_{y} \tilde{w}+C \nabla_{y} \pi=0, \quad \nabla_{y} \cdot \tilde{w}=0, \\
& \frac{1}{2}\left\langle\tilde{w} \cdot C^{-1} \tilde{w}\right\rangle=1, \\
& \tilde{w} y \text {-periodic in } Y \text { and } Q \text {-invariant. }
\end{aligned}
$$

Note that $w_{0}^{*}$ satisfies (1.31) with $C=I$. We assume that there exist solutions $w(y, C)$ of (1.31) which depend continuously on $C$. In this case, we obtain a reduced model, in which the dependence on $q$ of the closure functions is explicit:

$$
\begin{aligned}
& u_{, t}+u . \nabla u+\nabla p+\varepsilon^{2 / 3} \nabla .(q R)=0, \quad \nabla . u=0, \\
& q_{, t}+u . \nabla q+q(R: \nabla u+\mu \psi)=0, \\
& a_{, t}+u . \nabla a=0, \\
& u(x, 0)=u_{0}(x), \quad q(x, 0)=q_{0}(x), \quad a(x, 0)=x .
\end{aligned}
$$

The new closure functions $R$ and $\psi$ depend only on $G$, as follows:

$$
\begin{aligned}
& R(G)=G^{-T} \tilde{R}(C) G^{-1}, \quad \tilde{R}_{i j}(C)=\left\langle\tilde{w}_{i}(C) \tilde{w}_{j}(C)\right\rangle, \\
& \psi(G)=\psi(C)=C_{i j}^{-1}\left\langle\frac{\partial \tilde{w}_{i}(C)}{\partial y_{k}} \frac{\partial \tilde{w}_{j}(C)}{\partial y_{l}}\right\rangle C_{k l} .
\end{aligned}
$$

The existence of solutions of (1.31) which depend continuously on $C$ is an hypothesis which depends on how this equation is interpreted. We shall test it from a numerical point of view in $\S 2$.

1.3. Eddy viscosity terms. We have seen in the Introduction that the Reynolds tensor $R$ of our model seems not to be a diffusion tensor. However, the model contains 
eddy diffusion terms. To find them, we must extend the equations to include higher order terms. A lengthy analysis leads to the following equations (see [7]):

$$
\begin{aligned}
& u_{, t}+u . \nabla u+\nabla p+\nabla \cdot(k R)+\varepsilon \nabla \cdot(\sqrt{k} A: \nabla u)=0, \\
& \nabla . u=0, \\
& k_{, t}+u . \nabla k+k(R: \nabla u+\mu \psi)+\varepsilon^{2 / 3} \nabla \cdot(\sqrt{k} V: \nabla k)=0, \\
& a_{, t}+u . \nabla a=0, \\
& u(x, 0)=u_{0}(x), \quad k(x, 0)=\varepsilon^{2 / 3} q_{0}(x), \quad a(x, 0)=x .
\end{aligned}
$$

Here $A, V$ are $(3 \times 3)^{2}$ and $3 \times 3$ tensors, respectively, defined through a canonical linear microstructure problem. System (1.35) is written in function of the kinetic energy of the microstructure $k=\varepsilon^{2 / 3} q$.

A tabulation for $A$ was obtained at first when $C=I$. The signs show that the extra terms in (1.35) behave like eddy viscosity

$$
A: \nabla u \simeq-\frac{4}{9}\left(\nabla u+\nabla u^{T}\right)
$$

Also, numerical experiments show that a reasonable approximation of the diffusion term in $(1.35 \mathrm{c})$ is

$$
\nabla \cdot(\sqrt{k} V . \nabla k) \simeq-\frac{4}{6} \Delta\left(k^{3 / 2}\right) .
$$

The model given by (1.35)-(1.37) is the one which shall be considered in the remaining sections. In particular, it shall be used in the numerical experiments.

2. Computation of the closure functions. To test the model (1.35) numerically, we need to know the closure terms $R$ and $\psi$ as functions of $G$. Thus, we need to solve the microstructure problem (1.31) with the matrix $C$ as a parameter: we present in this section a numerical solution of (1.31) by least squares and its application to the tabulation of $R$ and $\psi$.

2.1. Computation of the canonical microstructure fluctuations. The numerical solution of the microstructure problem (1.31) has to be done very carefully: this problem involves a generalized stationary Euler equation and a quadratic constraint. As has been shown in [12], (1.31) does not have a unique solution. It is thus necessary to fix a solution in some way. Moreover, the fact that we are looking for periodic solutions excludes the use of methods for transport problems (the method of characteristics, for example).

We shall solve (1.31) by least squares. This method has been used successfully to regularize problems of fluid dynamics (see [4]). It seems to be well adapted to our problem. Nevertheless, this method can give unphysical solutions in some cases. Such solutions appear, for example, in the computation of transonic flows. In this case, the approximate solution contains a nonphysical shock if the entropy condition is not taken into account (see [4]). In our case, additional conditions of physical significance are not known for the perturbation $\tilde{w}$. Thus, the least squares generation of the microstructure may fail to produce acceptable solutions from the physical point of view and should be considered as tentative at present.

For a given nonsingular matrix $C$, let us consider the equation appearing in (1.31) (all the derivatives are taken with respect to the variable $y$ ):

$\tilde{w} . \nabla \tilde{w}+C \nabla \tilde{\pi}=0, \quad \nabla \cdot \tilde{w}=0$ in $Y, \quad \tilde{w} y$-periodic in $Y$ and $Q$-invariant.

For any given trial solution $\tilde{w}$ of $(2.1)$, let us define its associated error $\tilde{\xi}(\tilde{w})$ by

$$
-\Delta \tilde{\xi}=C^{-1} \nabla \cdot(\tilde{w} \otimes \tilde{w})+\nabla \tilde{p}, \quad\langle\tilde{\xi}\rangle=0, \quad \tilde{\xi} y \text {-periodic in } Y,
$$


where $\tilde{w} \otimes \tilde{w}$ is the $3 \times 3$ tensor of components $\tilde{w}_{i} \tilde{w}_{j}$, and $\tilde{p}$ is the associated pressure, defined by

$$
-\nabla \cdot(C \nabla \tilde{p})=\nabla \cdot(\tilde{w} \cdot \nabla \tilde{w}) ; \quad\langle\tilde{p}\rangle=0, \quad \tilde{p} y \text {-periodic in } Y .
$$

If $\tilde{w}$ is smooth enough (for example, differentiable with continuous derivatives), then $\tilde{w}$ solves $(2.1)$ if and only if $\tilde{\xi}(\tilde{w})=0$ (see [1]).

Then, the problem (2.1) is equivalent to

$$
\min _{\tilde{w} \in V}\|\tilde{\xi}(\tilde{w})\|^{2}=0
$$

where $V$ is an appropriate space of periodic $Q$-invariant vector fields, and $\|\tilde{\xi}\|$ denotes any appropriate norm for $\tilde{\xi}$. If we consider the existence results for the Navier-Stokes equations, it seems convenient to take a subspace of $L^{4}(Y)$ as space $V$ (see [21]).

We have not taken into account the condition $\frac{1}{2}\left\langle\tilde{w} \cdot C^{-1} \tilde{w}\right\rangle=1$ in the previous discussion. It can be easily satisfied if we get a nonzero solution of (2.1) $\tilde{w}$ since $\lambda \tilde{w}$ is also solution of (2.1) for every $\lambda \in \mathbb{R}$.

The discretization of the formulation (2.2)-(2.4) has been implemented by the finite element method, as follows: let $\mathscr{T}_{h}$ be a triangulation of the cube $Y$ by tetrahedra. Let us define the discrete space for pressures $Q_{h}$ by

$$
Q_{h}=\left\{q_{h} \in C^{0}(\bar{Y}) /\left.q_{h}\right|_{T} \in \mathbb{P}_{1}, \forall T \in \mathscr{T}_{h},\left\langle q_{h}\right\rangle=0, q_{h} \text {-periodic in } Y\right\}
$$

where $C^{0}(\bar{Y})$ is the space of continuous functions on $\bar{Y}$, and $\mathbb{P}_{1}$ the one of linear functions. The discrete space for velocities is

$$
V_{h}=\left\{\tilde{w}_{h} \in\left[Q_{h / 2}\right]^{3} / \tilde{w}_{h} \text { is } Q \text {-invariant }\right\} .
$$

Here, $Q_{h / 2}$ is the space defined in (2.5), corresponding to a triangulation $\mathscr{T}_{h / 2}$ obtained from $\mathscr{T}_{h}$ by subdividing each tetrahedron of $\mathscr{T}_{h}$ into eight tetrahedra.

The discrete spaces have been chosen like this in order to verify the Brezzi-Babuska inf sup condition (see Glowinski and Pironneau [10]). This is a necessary condition for the convergence of the finite element method applied to the Stokes equation. Note also that $V_{h} \subset\left[H^{1}(Y)\right]^{3} \subset\left[L^{4}(Y)\right]^{3}$.

For $\tilde{w}_{h} \in V_{h}$, let us define its residual associated error $\tilde{\xi}_{h}$ by

$$
\begin{aligned}
& \int_{Y} \nabla \tilde{\xi}_{h}: \nabla \tilde{u}_{h} d y=-\int_{Y} C^{-1}\left(\tilde{w}_{h} \otimes \tilde{w}_{h}\right): \nabla \tilde{u}_{h} d y+\int_{Y} \nabla \tilde{p}_{h} . \tilde{u}_{h} d y \quad \forall \tilde{u}_{h} \in\left[Q_{h / 2}\right]^{3}, \\
& \tilde{\xi}_{h} \in\left[Q_{h / 2}\right]^{3} .
\end{aligned}
$$

where the corresponding pressure $\tilde{p}_{h}$ is defined by

$$
\begin{aligned}
& \int_{Y} C \nabla \tilde{p}_{h} \cdot \nabla \tilde{q}_{h} d y=-\int_{Y} \tilde{w}_{h} \cdot \nabla \tilde{w}_{h} \cdot \nabla \tilde{q}_{h} d y \quad \forall \tilde{q}_{h} \in Q_{h}, \\
& \tilde{p}_{h} \in Q_{h} .
\end{aligned}
$$

The problems (2.7) and (2.8) are of elliptic type and have unique solutions. A discrete equivalent of (2.4) which seems to be appropriate is

$$
\min _{\tilde{w}_{h} \in \tilde{V}_{h}} E\left(\tilde{w}_{h}\right), \quad E\left(\tilde{w}_{h}\right)=\int_{Y}\left|\nabla \tilde{\xi}_{h}\right|^{2} d y .
$$

We are thus minimizing the $H^{-1}(Y)$ norm of the right-hand side of (2.2). However, the formulation (2.7)-(2.9) does not eliminate the solution zero. Since the cost function $E$ is homogeneous, it is better to replace (2.9) by

$$
\min _{\tilde{w}_{h} \in \tilde{V}_{h}} J\left(\tilde{w}_{h}\right), \quad J\left(\tilde{w}_{h}\right)=E\left(\frac{\tilde{w}_{h}}{\left\langle\tilde{w}_{h} \cdot \tilde{w}_{h}\right\rangle^{1 / 2}}\right) .
$$


This is equivalent to solving (2.9) on the unit sphere of $L^{2}(Y)$. Problem (2.10) has been solved by the conjugate gradient method. Note that we have to assume the existence of isolated solutions of $(1.31)$, belonging to $\left[L^{4}(Y)\right]^{3}$, for example.

The triangulation $\mathscr{T}_{h}$ is symmetric about the three coordinate planes. It preserves the $Q$-invariance of $\tilde{w}_{h}$ in the conjugate gradient algorithm. This means that we can replace $\tilde{V}_{h}$ by $\left[Q_{h / 2}\right]^{3}$ in (2.9) if the initialization of the minimizing process is $Q$ invariant.

The problems (2.7) and (2.8) have been solved by using the Fast Fourier Transform and a fixed point algorithm. This provides a rapid inversion of the elliptic operators appearing in (2.7), (2.8) (see [6]). The numerical computation has been carried out on a CRAY 1 computer with a $16 \times 16 \times 16$ grid for velocities. It takes approximately $700 \mathrm{~s}$ of CPU for 20 iterations of the conjugate gradient. This corresponds to a descent of the relative cost from 1 to $\sim 10^{-3}$.

2.2. Tabulations. We will consider one-dimensional mean flows as follows:

$$
u(x, t)=\left(u_{1}\left(x_{3}, t\right), 0,0\right)^{T} .
$$

These flows will be studied in $\S \S 3$ and 4 . In this case,

$$
\nabla a=\left[\begin{array}{ccc}
1+\alpha^{2} & 0 & \alpha \\
0 & 1 & 0 \\
\alpha & 0 & 1
\end{array}\right] \text { with } \alpha=-\int_{0}^{t} u_{1, \xi}(\xi, s) d s
$$

From the $Q$-invariance of $\tilde{w}$, the Reynolds tensor $R$, defined by (1.27), has the following structure:

$$
R=R(\alpha)=\left[\begin{array}{ccc}
R_{11} & 0 & R_{13} \\
0 & R_{22} & 0 \\
R_{13} & 0 & R_{23}
\end{array}\right]
$$

Figure 1 shows the computed $\rho=R_{13}$ as a function of the parameter $\alpha$. Only nonnegative values of $\alpha$ are considered because $\rho(-\alpha)=-\rho(\alpha)$ from the $Q$-invariance. It is a regular function away from $\alpha=0$. Near the origin, it has a very high gradient.

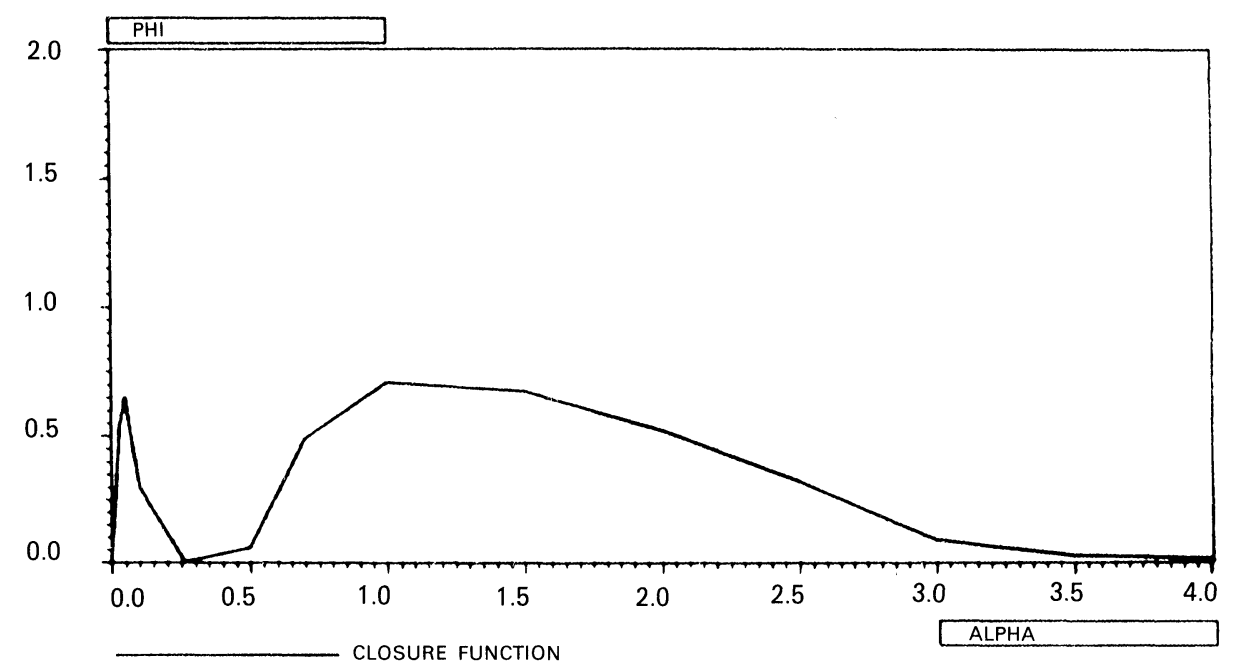

FIG. 1. Computed closure function $\rho(\alpha)$. It is a smooth function with a high gradient at $\alpha=0$. Notice that $\rho^{\prime}(0)>0$. 
3. Numerical experiments. In this section, the reduced model (1.35) is tested by three-dimensional Poiseuille flows with one-dimensional mean. The test is made by a comparison between the direct simulation and the simulation of the model. Good agreement is obtained in the behavior of both the mean velocity field and the kinetic energy of the microstructure.

Let us consider the Navier-Stokes equations for incompressible flows with kinematic viscosity $\nu$ :

$$
u_{, t}+u . \nabla u+\nabla p-\nu \Delta u=0, \quad \nabla . u=0,
$$

in the domain $\Omega=\mathbb{B} \times \mathbb{R} \times] 0,2\left[\right.$ for time $t>0$. We are interested in $x_{1}-x_{2}$ periodic solutions of the Poiseuille flow. For given $\alpha_{1}>0, \alpha_{2}>0$ it satisfies

$$
u\left(x_{1}+\frac{2 \pi}{\alpha_{1}} i, x_{2}+\frac{2 \pi}{\alpha_{2}} j, x_{3} ; t\right)=u\left(x_{1}, x_{2}, x_{3} ; t\right) \quad \forall x_{3} \in[0,2] \quad \forall t \geqq 0,
$$

for any integer numbers $i, j$.

$$
u\left(x_{1}, x_{2}, x_{3} ; t\right)=0 \quad \text { at } x_{3}=0 \quad \text { and } \quad x_{3}=2, \quad \forall t \geqq 0 .
$$

Our purpose is to test the model (1.35) with such a flow.

We will compare the solution of (3.1)-(3.3) with an initialization in microstructure, as (1.30), and the solution of (1.35) with the corresponding initialization. We have used a spectral method to solve numerically (3.1)-(3.3), developed by Orszag and Patera [15]. The velocity field is discretized by Fourier series about the $x_{1}, x_{2}$ variables, and by Chebyshev's expansion about the $x_{3}$ variable, as follows:

$$
\begin{aligned}
& u\left(x_{1}, x_{2}, x_{3} ; t\right)=\sum_{|n| \leqq N} \sum_{|m| \leqq M} v_{n m}\left(x_{3}, t\right) e^{i\left(\alpha_{1} n x_{1}+\alpha_{2} m x_{2}\right)} \\
& \text { with } v_{n m}\left(x_{3}, t\right)=\sum_{p=0}^{P} v_{n m p}(t) T_{p}\left(x_{3}\right) .
\end{aligned}
$$

Here, $T_{p}$ is the $p$ th Chebyshev polynomial, defined by

$$
T_{p}(\cos \theta)=\cos (p \theta) .
$$

This is a robust code, which has been used in the study of the stability of the Poiseuille flow with very good results. Moreover, it is highly vectorized (see [15]). The discretization (3.4) allows us to take initializations in microstructure easily. Let us consider the following initialization of (3.1)-(3.3), for a given integer $L \leqq \min (N, M)$,

$$
u(x, 0)=u_{0}(x)+\operatorname{Re}\left[v\left(x_{3}\right) e^{i\left(\alpha_{1} x_{1}+\alpha_{2} x_{2}\right) L}\right],
$$

where $u_{0}(x)$ is the basic Poiseuille flow

$$
u_{0}(x)=\left(1-\left(1-x_{3}\right)^{2}, 0,0\right)^{T},
$$

and $v\left(x_{3}\right)$ is an arbitrary function such that $\nabla \cdot u(x, 0)=0$. The initialization corresponds to the following perturbation in microstructure

$$
\varepsilon^{1 / 3} w_{0}(y, x)=\operatorname{Re}\left[v\left(x_{3}\right) e^{i\left(\alpha_{1} y_{1}+\alpha_{2} y_{2}\right)}\right] \quad \text { with } \varepsilon=1 / L .
$$

We have taken $v\left(x_{3}\right)=0$ near the boundaries $x_{3}=0$ and $x_{3}=2$ because our model is not concerned with turbulent boundary layers. For such initializations, the corresponding initial conditions of the system (1.35) are

$$
u_{0}(x, 0)=\left(1-\left(1-x_{3}\right)^{2}, 0,0\right)^{T}, \quad k(x, 0)=\frac{1}{2}\left|v\left(x_{3}\right)\right|^{2} .
$$


These conditions depend only upon $x_{3}$. As the boundary conditions (3.3) are homogeneous, all quantities appearing in (1.35) will depend only on $x_{3}$ and $t$. Then the Reynolds tensor $R$ has the structure given by (2.13). Consequently, the model (1.35) reduces to

$$
\begin{aligned}
& u_{1, t}+(k \rho)_{, \xi}-\frac{4}{9} \varepsilon\left(\sqrt{k} u_{1, \xi}\right)_{, \xi}=0, \\
& k_{, \tau}+k\left(\rho u_{1, \xi}+\mu \psi\right)-\frac{4}{9} \varepsilon^{2 / 3}\left(\sqrt{k} k_{, \xi}\right)_{, \xi}=0,
\end{aligned}
$$

where $\rho=R_{13}$ and $\psi$ depend only on the parameter $\alpha$ defined in (2.12) and $\mu=\nu \varepsilon^{-2}$. The boundary conditions corresponding to (3.8), (3.9) are

$$
u_{1}(\xi= \pm 2)=0, \quad k(\xi= \pm 2)=0 .
$$

Problem (3.8)-(3.10) is discretized by a finite difference method. The space discretization is uniform, with 65 grid points so as to have good resolution near the boundaries. The direct solution of (3.1)-(3.3) with the initial conditions (3.5) has also been carried out on a CRAY 1 computer by using a $32 \times 32 \times 33$ grid. This allows us to have $N=M=16$ and $P=32$ in (3.4). We have taken $\varepsilon=0.1$ (i.e., $L=10$ ). This is a good choice because $\varepsilon$ is relatively small but the corresponding wave number $L$ is not too close to the maximum wave number taken into account by the discretization $(N=M=16)$. The values for $\alpha_{1}$ and $\alpha_{2}$ are rather arbitrary. Also, $\nu$ must be of order $\varepsilon^{2}$. We have taken $\nu=1 / 4000, \alpha_{1}=\alpha_{2}=1.32$.

To test the results, we must compare the functions $\left(u_{1}, k\right)$ defined by $(3.8)-(3.10)$ with the corresponding functions defined from the solution of (3.1)-(3.3), with the initialization (3.5). It is important to define suitably the equivalent $k^{*}$ of the kinetic energy of the microstructure $k$. It has been defined by summing all the kinetic energies associated to wave numbers $n$ and $m$ greater than five in (3.4)

$$
k^{*}(\xi, t)=\sum_{|n| \geqq 6} \sum_{|m| \geqq 6}\left|v_{n m}(\xi, t)\right|^{2} .
$$

This choice of $k^{*}$ is made for the following reason: in the initialization (3.5), the energy of the wave number $n=m=L$ is $k(x, 0)=k^{*}(x, 0)=\frac{1}{2}\left|v\left(x_{3}\right)\right|^{2}$. Also, the energy corresponding to the other wave numbers greater than 1 is zero. However, when the time increases, there is a transfer of energy from the wave number $n=m=L$ to the other wave numbers. This can be seen in Fig. 2. If we define $k^{*} \operatorname{simply}$ as $k^{*}(x, t)=$ $\left|v_{L L}\left(x_{3}, t\right)\right|^{2}$, there is some kinetic energy corresponding to the microstructures which is not being taken into account. In fact, the wave number $n=m=6$ represents in (3.11) the lower limit of the microstructure scales.

Let us note that our comparison is valid only if there is a spectral gap in the kinetic energy corresponding to the direct simulation (3.1)-(3.3). We can expect that this happens during a time of order $\varepsilon^{-2 / 3}$. This hypothesis is confirmed by the experiments. For example, in the spectrum of Fig. 2, corresponding to $\varepsilon=0.1$, there is a "gap" for $t \leqq 3$.

Figures 3 and 4 show the results of both simulations considered, corresponding to parabolic $v$ in (3.5). The time scales are similar, and the shapes of velocity and kinetic energy of the microstructure are in good agreement. Several experiments have been done, by changing the shape of $v$, and these result in comparable agreement.

Differences between the profiles for kinetic energies can be observed in Figs. 4(a) and $4(\mathrm{~b})$. These differences are probably due to the fact that the tabulations of the closure terms appearing in (3.9) are only approximate. Another reason which can partially justify those differences is that the model equations (3.9) are truncated at the second order in $\varepsilon$. 


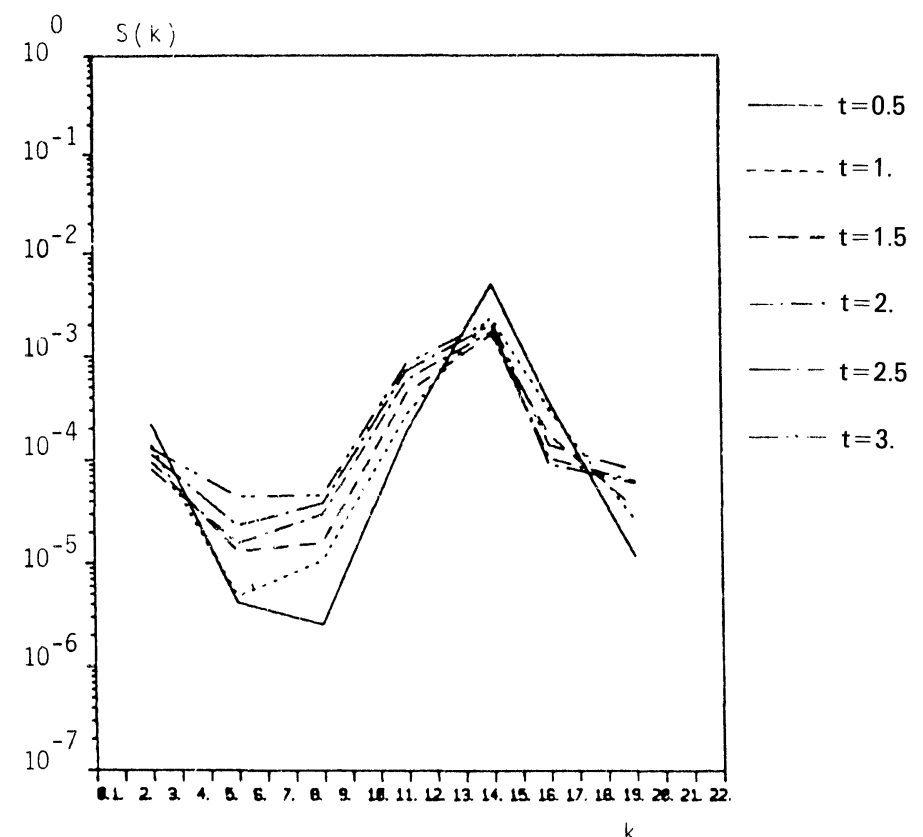

FIG. 2. Typical energy spectrum. The values represented are

$$
S(k, t)=\sum_{k-1 \leqq \sqrt{n}^{2}+m^{2} \leqq k} \int_{0}^{2}\left|v_{n m}\left(x_{3}, t\right)\right|^{2} d x_{3} .
$$

We may say that, based on our numerical experiments, the model considered correctly simulates the interactions between large and small structures when there is a spectral gap and for short times.

It is reasonable to expect that the model is valid for longer times if the initial gap between the spectra of large and small structures is larger. To simulate this situation, we need values of $\varepsilon$ smaller than the one considered here $(\varepsilon=0.1)$. But this would require a computer with a much larger memory than the one used (a CRAY 1-S).

4. A wave equation for turbulence modeling. In this section we shall test numerically the reduced equations of model (0.7)-(0.9). Our purpose is to see if the Reynolds tensor $R$ of our model has a hyperbolic character.

Let us consider plane Poiseuille flow, as in $\S 3$. If we neglect all viscous effects, the model (3.9) reduces to

$$
u_{1, t}+(k \rho)_{, \xi}=0, \quad k_{, t}+k \rho u_{1, \xi}=0 .
$$

As is stated in [12], this equation can be written as follows:

$$
\begin{aligned}
& V_{, t t}+\left[k(\xi, 0) e^{\phi\left(-V_{, \xi}\right)} \rho\left(-V_{, \xi}\right)\right]_{, \xi}=0, \\
& q(\xi, t)=q(\xi, 0) e^{\phi(\alpha(\xi, t))}, \\
& V(\xi, t)=\int_{0}^{t} u_{1}(\xi, s) d s, \quad \phi(\alpha)=\int_{0}^{\alpha} \rho(\beta) d \beta .
\end{aligned}
$$

The character of (4.2) can be studied by linearization. Symmetries imply $\rho(0)=0$. So, if $\rho^{\prime}(0) \neq 0$, we get

$$
V_{, t t}-k_{0} \rho^{\prime}(0) V_{, \xi \xi}=0 .
$$

From Fig. 1, we see that $\rho^{\prime}(0)>0$. In this case, (4.5) is a wave equation. 


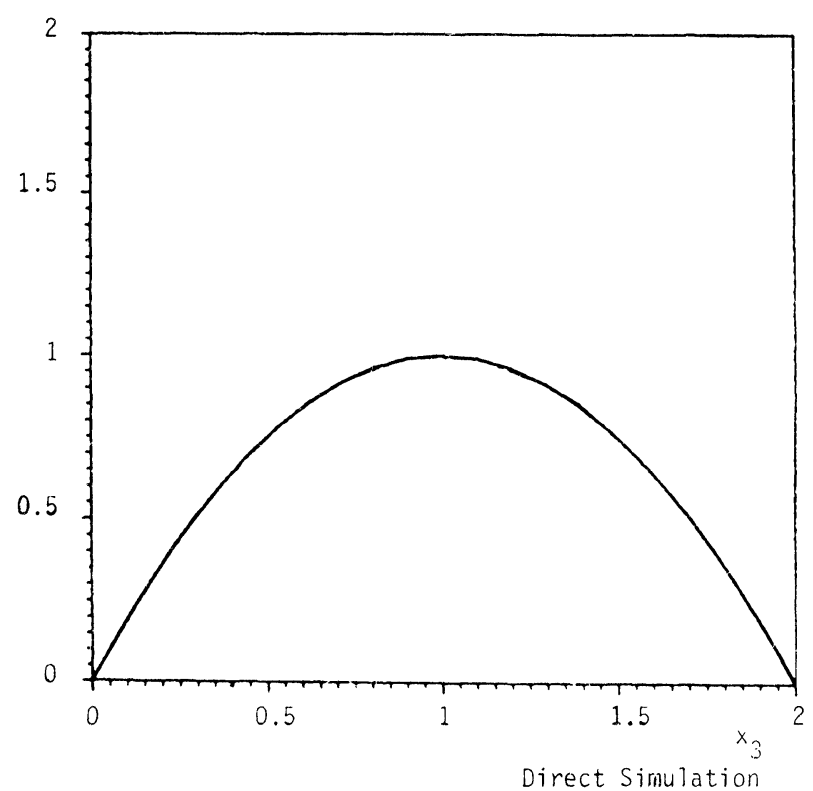

(a)

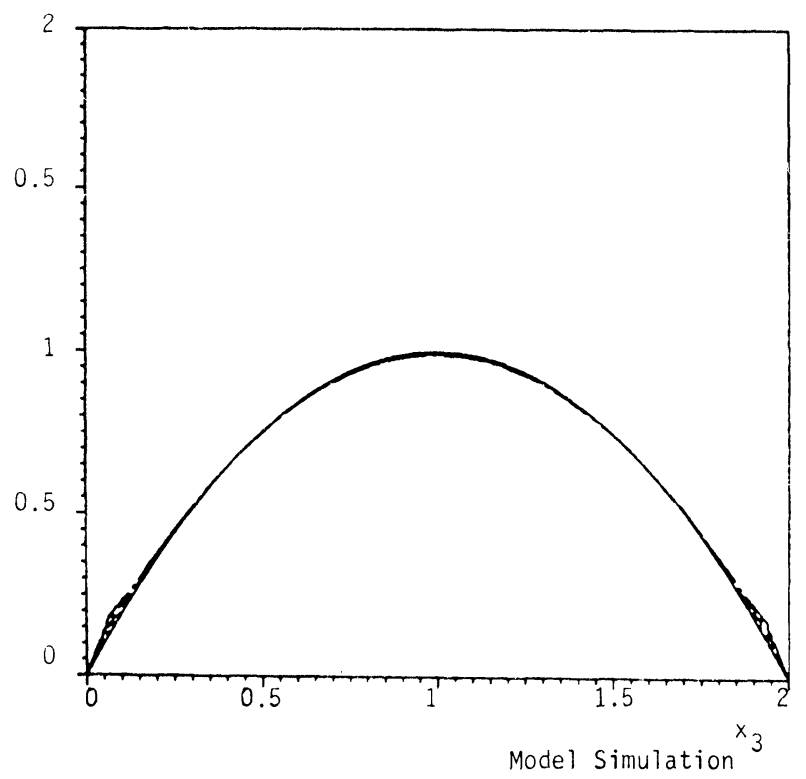

(b)

FIG. 3(a). First component of the mean velocity field. Corresponds to the direct simulation (3.1)-(3.3) and to parabolic $v$ in (3.7). Times are as in Fig. 2, less 0.5. (b). Function $u_{1}$. Corresponds to the reduced model (3.8)-(3.9). Times are as in Fig. 2, less 0.5. 


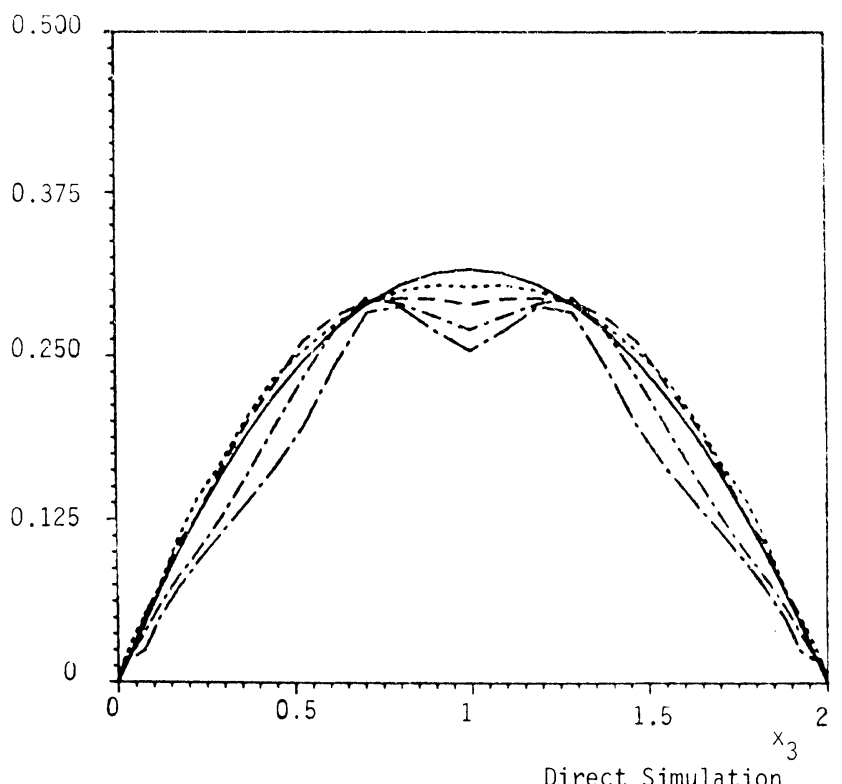

(a)

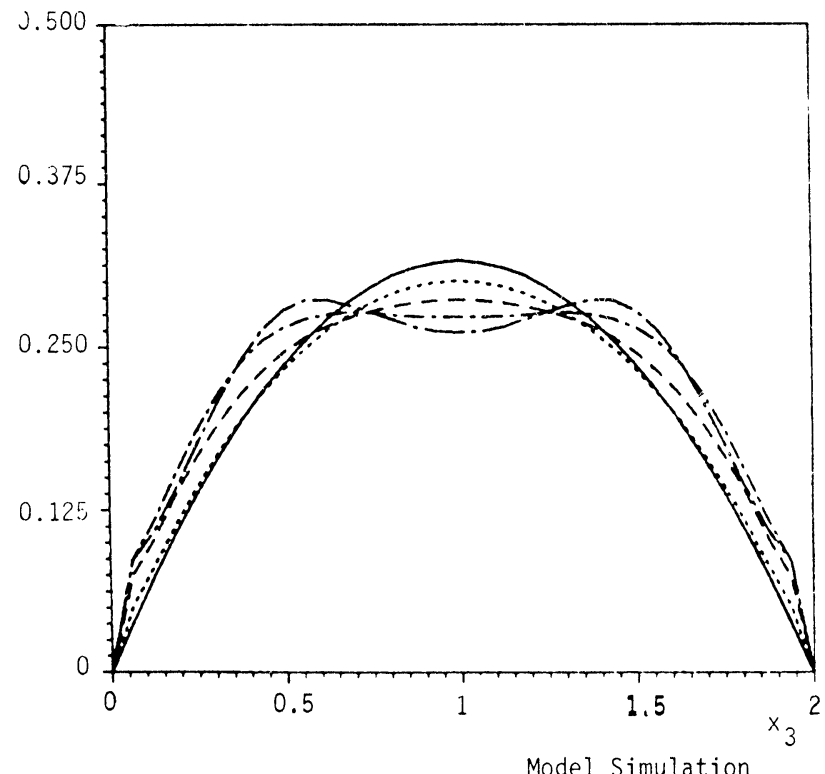

(b)

FIG. 4(a). Mean kinetic energy of the microstructures $k^{*}$. Corresponds to the direct simulation (3.1)-(3.3). It is defined by (3.11). Times are as in Fig. 2, less 0.5. (b). Mean kinetic energy of the microstructures $k$. Corresponds to the reduced model (3.8)-(3.9). Times are as in Fig. 2, less 0.5. 
A numerical analysis of this problem has been carried out by direct simulation. We have solved (3.1)-(3.3) with initializations given by (3.5). An oscillatory behavior has been seen clearly on a particular function: the rate of exponential decay of the kinetic energy of the microstructure $C(t)$, defined by

$$
C(t)=\frac{d}{d t}\left[\log \bar{e}_{m}(t)\right], \quad \bar{e}_{m}(t)=\int_{0}^{2}\left|v_{L L}(\xi, t)\right|^{2} d \xi .
$$

Here $\bar{e}_{m}$ is the mean kinetic energy of the microstructure. The function $C(t)$ is showed in Fig. 5 for $\bar{e}_{m}(0)=0.1$, and $L=10$. The oscillations are clearly seen here. Moreover, (4.5) gives an estimate for the period $\tau$ of those oscillations which is, approximately,

$$
\varepsilon=K / \sqrt{\bar{e}_{m}(0)} \text { with } K \text { constant. }
$$

This prediction has been substantiated by the direct simulation. The array in Table 1 shows the computed and predicted values of $\tau$ in several cases, with relatively small

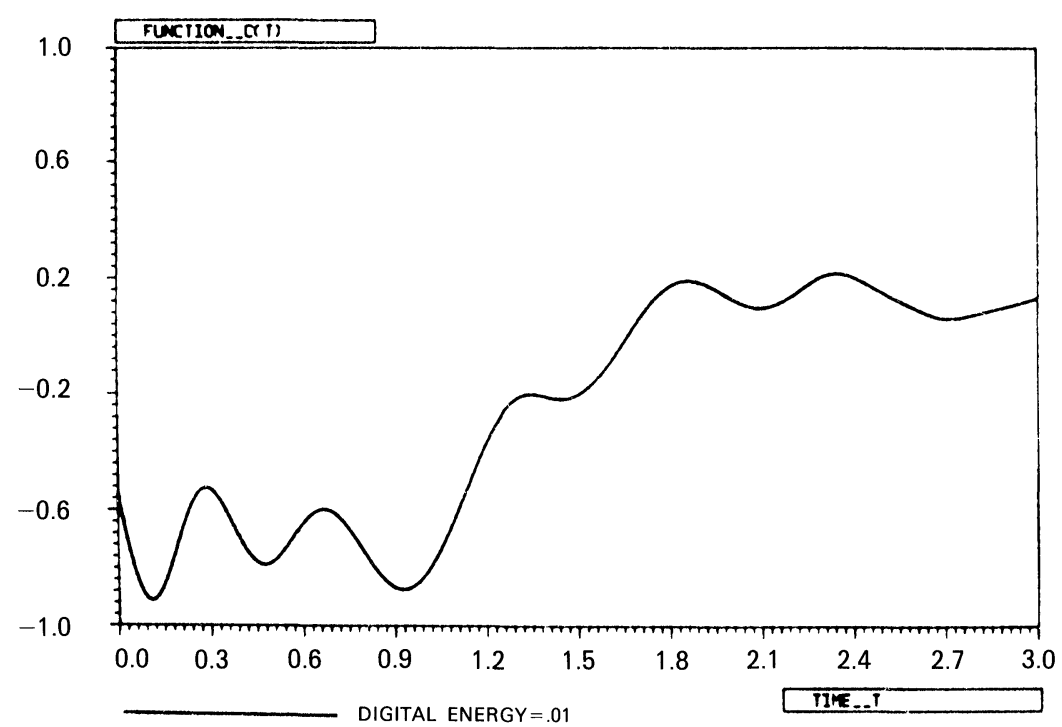

FIG. 5. Rate of exponential growth of the mean microstructure kinetic energy $C(t)$ defined by (4.7), corresponding to $\bar{e}_{m}(0)=0.01$. The oscillations are well demonstrated, with almost constant period for $t<1$. For $t>1$, there is a progressive relaxation of the oscillations due to the "filling" of the energy spectrum (see Fig. 2). This relaxation has been observed in all the cases considered.

TABLE 1

Comparison between the computed value of the period of the oscillations and the predictions. The case $\bar{e}_{m}(0)=0.01$ has been used to calculate the constant $K$ in $(4.7)$. The value taken for the period in the direct simulation has been the time between the two first minimum of $C(t)$. Notice that the periods are roughly well predicted, the errors being under $25 \%$.

\begin{tabular}{c|l|l|c|c}
\hline$e(0)$ & $\begin{array}{c}\tau_{m} \\
\text { measured }\end{array}$ & $\begin{array}{c}\tau_{p} \\
\text { predicted }\end{array}$ & $\begin{array}{c}\text { Relative } \\
\text { error \% }\end{array}$ & $\begin{array}{c}\text { Absolute } \\
\text { error }\end{array}$ \\
\hline 0.2 & 0.105 & 0.082 & 21.9 & 0.023 \\
0.1 & 0.1475 & 0.116 & 18.2 & 0.031 \\
0.04 & 0.22 & 0.184 & 16.4 & 0.036 \\
0.01 & 0.3675 & & \\
\hline$K=0.03675$ & \multicolumn{2}{|c}{} \\
\hline
\end{tabular}


errors. The energy lost by the scale corresponding to the wave number $n=m=L$ from $t=0$ to $t=\tau$ is very small so that the energy corresponding to the other scales is not relevant in the definition of $\bar{e}_{m}$.

Let us remark that the computed value of $\rho^{\prime}(0)$ for $\varepsilon=0.1$, by the direct simulation, is of the order of $10^{5}$. It agrees with the high value of $\rho^{\prime}(0)$ which has been computed in the tabulations (see Fig. 1). Note also that (4.1)-(4.5) are deduced from the model introduced in $\S 1$ which applies to rapid oscillations of small amplitude. Nevertheless, the same equations may be formally derived from the original model of [12] (0.7)-(0.9). In the numerical experiments presented in this section, only oscillations of small amplitude are considered to avoid numerical instability.

The results presented above provide a good indication that oscillatory behavior is an inherent property of the interaction between large and small scale structures, when dissipative effects are negligible.

Acknowledgments. The author thanks C. Begue and A. Patera for letting him use their FORTRAN codes, and also P. Ortegom, G. Papanicolaou, and O. Pironneau for several discussions and comments about this work.

\section{REFERENCES}

[1] C. Begue. Simulation de la turbulence par méthode d'homogénéisation, Third Cycle Thesis, Univ. Paris VI, December 1983.

[2] C. Begue, T. Chacon, D. Mclaughlin, G. Papanicolaou, and O. Pironneau Convection of Microstructures II, in Proc. Sixth International Symposium on Computing Methods in Applied Sciences and Engineering, R. Glowinski and J. L. Lions eds., Versailles, France, December 1983, North-Holland, Amsterdam, 1984.

[3] A. Bensoussan, J. L. Lions, And G. Papanicolaou, Asymptotic Methods for Periodic Structures, North-Holland, Amsterdam, 1978.

[4] M. O. Bristeau, R. Glowinski, J. Periaux, P. Perrier, and O. Pironneau, On the numerical solution of nonlinear problems in fluid dynamics by least squares and finite element methods (I). Least squares formulation and conjugate gradient solution of the continuous problems, Comput. Methods Appl. Mech. Engrg., 17/18 (1979), pp. 619-657.

[5] Ph. Ciarlet, Lectures on Three-Dimensional Elasticity, Tata Institute Lectures on Mathematics, Springer-Verlag, Berlin, 1983.

[6] T. Chacon, Contribucion al estudio del modelo M.P.P. de turbulencia, Thesis, Univ. Sevilla, Spain, September 1984.

[7] - Etude d'un modèle pour la convection des microstructures, Third Cycle Thesis, Univ. Paris VI, April 1985.

[8] T. Chacon and O. Pironneau, On the mathematical foundations of the $k-\varepsilon$ turbulent model, in Vistas in Applied Mathematics, A. V. Balakrishnan, A. A. Dorodnitsyn and J. L. Lions eds., Springer-Verlag, New York, 1986, pp. 44-56.

[9] U. Frisch, Z. S. She, And O. Thual, On the Elastic Behavior of Turbulence, in Proc. Workshop on Numerical Simulation of Turbulence, Nice, France, December 1984, U. Frisch, J. B. Keller, G. Papanicolaou and O. Pironneau eds., Springer-Verlag, Berlin, 1985.

[10] R. Glowinski And O. Pironneau, On numerical methods for the Stokes problem, Energy Methods in Finite Element Analysis, Chapter 13, R. Glowinski, E. Y. Rodin and O. C. Zienkiewicz eds., John Wiley, Chichester, 1979, pp. 243-264.

[11] B. E. Launder And D. B. Spalding, Mathematical Models of Turbulence, Academic Press, New York, 1972.

[12] D. Mclaughlin, G. Papapanicolaou, and O. Pironneau, Convection of microstructures, Siam J. Appl. Math., 45 (1985), pp. 780-797.

[13] B. Mercier, Analyse numérique des méthodes spectrales, Note CEA-N-2278, June 1981.

[14] P. J. Olver, A nonlinear Hamiltonian system structure for the Euler equations, J. Math. Anal. Appl., 89 (1982), pp. 233-250.

[15] S. A. Orszag And A. T. Patera, Secondary instability of wall-bounded shear flows, J. Fluid Mech., 128 (1983), pp. 347-385. 
[16] G. Papanicolaou And O. Pironneau, On the asymptotic behaviour of motion in random flows, in Stochastic Nonlinear Systems, Arnold and Lefeever, eds., Springer-Verlag, Berlin, 1981.

[17] P. Perrier and O. Pironneau, Subgrid turbulence modelling by homogenization, Math. Model., 2 (1981), pp. 295-317.

[18] A. Pouquet, U. Frisch, And J. P. Chollet, Turbulence with a spectral gap, Phys. Fluids, 26 (1983), pp. $877-880$.

[19] V. SCHUMAnN, Subgrid scale model for finite difference simulations of turbulent flows in plane channel and annuli, J. Comput. Phys., 18 (1975), pp. 376-404.

[20] L. TARTAR, Remarks on oscillations and Stoke's equations, Proc. Workshop on Numerical Simulation of Turbulence, Nice, France, December 1984, U. Frisch, J. B. Keller, G. Papanicolaou, and O. Pironneau eds., Springer-Verlag, Berlin, 1985.

[21] R. Temam, Navier-Stokes Equations, North-Holland, Amsterdam, 1977.

[22] P. L. VIOLET, On the modelling of turbulence heat and mass transfer for the computation of buoyancy affected flows, Proc. Internat. Conference on Numerical Methods in Laminar and Turbulent Flows, Venezia, 1981. 
Reproduced with permission of the copyright owner. Further reproduction prohibited without permission. 